\title{
Tablet Particle Crystal Dosage Form
}

National Cancer Institute

\section{Source}

National Cancer Institute. Tablet Particle Crystal Dosage Form. NCI Thesaurus. Code C69006.

A tablet composed of small fragments of singular, repeating units of that are systematically arranged over an evenly spaced lattice. 